\title{
False-Positive Clostridium difficile in Negative-Control Reactions Peak and Then Decrease with Repetitive Refrigeration of Immunoassay
}

\author{
Alexander Rodriguez-Palacios, ${ }^{1,2}$ Henry R. Stämpfli, ${ }^{3}$ and Yung-Fu Chang ${ }^{1}$ \\ ${ }^{1}$ Department of Population Medicine and Diagnostic Sciences, Cornell University, Ithaca, NY 14580, USA \\ ${ }^{2}$ Division of Gastroenterology and Liver Disease, Case Western Reserve University, Cleveland, OH 44106, USA \\ ${ }^{3}$ Department of Clinical Studies, University of Guelph, Guelph, ON, Canada N1G 2W1 \\ Correspondence should be addressed to Alexander Rodriguez-Palacios; axr503@case.edu
}

Received 14 May 2014; Accepted 25 June 2014; Published 11 September 2014

Academic Editor: Isabel Gómez

Copyright (C) 2014 Alexander Rodriguez-Palacios et al. This is an open access article distributed under the Creative Commons Attribution License, which permits unrestricted use, distribution, and reproduction in any medium, provided the original work is properly cited.

\begin{abstract}
Aberrant false-positive reactions in negative-controls during ELISA testing for Clostridium difficile indicated the potential for falsediagnoses. Experiments with 96 -well products showed a maximum peak of false-positive immunoassay reactions with the provided negative-control reagents after 5 refrigeration-to-room temperature cycles $(P<0.001)$, decreasing thereafter with additional refrigeration cycles. Because repetitive refrigeration causes a peak of false-positives, the use of single negative-controls per ELISA run might be insufficient to monitor aberrant preanalytical false-positives if immunoassays are subject to repetitive refrigeration.
\end{abstract}

\section{Introduction}

The diagnostic performance of ELISA immunoassays for the detection of toxins $\mathrm{A}$ and $\mathrm{B}$ of Clostridium difficile is still hampered by false-positive diagnoses [1-3]. Despite their recognized occurrence and the potential role of stool desorbing activity [4] and other host-associated factors [5], the mechanisms underlying false-positivity dependent on the immunoassays remain unclear. To prevent epidemiological bias, negative-controls must be used [6] to warn when falsepositives occur due to product failure.

As per most manufacturers, every commercial ELISA needs two controls, one positive and one negative. Laboratories often run samples in small batches especially when diagnostic demands are low. Under those circumstances, ELISAs in 96-well formats may be subject to multiple refrigerationto-room-temperature cycles (refrigeration cycles). Although manufacturers state that their 96-well products are stable if kept at constant refrigeration, this may not always be possible unless reagents are initially aliquoted and thereafter used onealiquot-at-a-time. To date, commercial products often do not provide information regarding how many refrigeration cycles are tolerable and their effect on test performance.

Recently, we observed aberrant false-positives reactions in negative-controls, in a laboratory with long-standing research and diagnostic capabilities for $C$. difficile [7-10]. The false-positives occurred progressively as a 96 -well format commercial immunoassay kit for the detection of $C$. difficile toxins A and B [11] was used to test small batches of fecal samples over time. Product handling was in accordance with product specifications [11]. Upon quality control examination of the product, 5 months prior to its expiration date, analysis indicated that the repetitive refrigeration of the ELISA wells could have been the cause of impaired performance. To date there are no available studies assessing the impact of repetitive refrigeration on false-positive performance in the negative commercial controls that are part of an ELISA kit. Here, we quantified the effect of repetitive refrigeration on falsepositivity of negative-controls using a 96-well ELISA product that instructs to bring to room temperature all refrigerated reagents (liquid and dry ELISA wells) every time the product is needed for testing $[12,13]$. 


\section{Materials and Methods}

To precisely quantify the hypothetical deleterious effect of refrigeration on the performance of negative-control reactions, a blinded two-factor experiment (number of refrigeration cycles, expiration status) was conducted with an ELISA product that instructed that all reagents should reach room temperature prior testing (Meridian Bioscience, Inc., Cincinnati, OH) $[12,13]$. One ELISA kit purchased from an authorized vendor, kept under stable refrigeration, and valid for testing with 6 months prior to the expiration date was used for testing. Two other Meridian kits with 3 and 15 months after their expiry dates were available to verify if falsepositives would occur after the product's expiry date. For each of the three kits tested, all available wells were randomly assigned to four groups ( $n=4-11$ wells/group), placed inside a $50 \mathrm{~mL}$ plastic tube with fifteen $0.5 \mathrm{~cm}$ perforations, and covered with aluminum foil to allow acclimation and protect from light. Each of the tubes was exposed to one of four conditions: 20, 10, 5, or 0 refrigeration cycles. Each cycle comprised alternate exposure to refrigeration $(2 \pm$ $\left.5 \mathrm{~h} ; 4^{\circ} \mathrm{C}\right)$ and room temperature $\left(1 \pm 0.2 \mathrm{~h}, 23^{\circ} \mathrm{C}\right.$, relative humidity of $70 \%)$. After 20 cycles, all wells $(n=105)$ were simultaneously tested using the corresponding liquid reagents, which were not subject to refrigeration changes. The negative-control reactions were performed using the provided negative-control commercial reagents, which are free of C. difficile toxins, following the manufacturer's instructions [12, 13]. To ensure homogeneous washing, prevent well-to-well spillage, and allow the systematic distribution of technical variability, random wells from all four groups were orderly alternated, leaving empty spaces in between, when placed on the 96-well washing tray. $\mathrm{OD}_{450}$ readings were used following the manufacturer's cutoff criteria to categorize reactions as positive or negative $[12,13]$.

\section{Results}

Analysis of $\mathrm{OD}_{450}$ data indicated that repetitive refrigeration had significant diagnostic deleterious effect on the performance in the negative-control reactions. Of primary concern, supporting the observation of aberrant positivity, there was a peak of false-positivity by the fifth refrigeration cycle $(P<$ 0.001 ), which gradually decreased over 15 additional cycles (Figure 1). Positive-controls performed as expected $\left(\mathrm{OD}_{450}>\right.$ 0.9 ; above 0.5 cutoff). As expected, none of the expired reagents provided any measurable reactivity indicating that false-positives are not random and that they do not occur in expired products.

\section{Discussion}

Appropriate negative-controls are fundamental in research and clinical practice [6], especially in small laboratory settings [2]. The present study quantified for the first time that repetitive refrigeration causes a peak of false-positives in the commercial ELISA negative-control reactions that should be negative because the reagents are free of $C$. difficile toxins. The cause of false-positivity is unclear but may be due to gradual

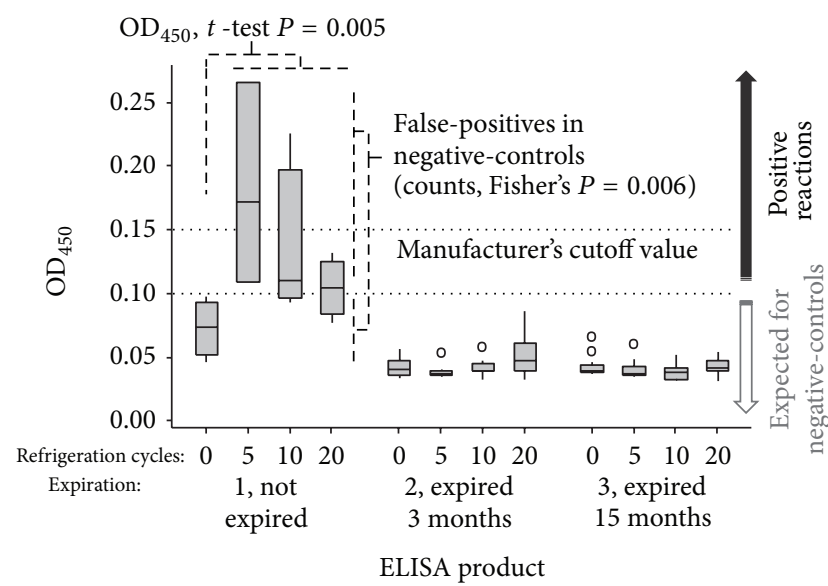

FIgURE 1: Refrigeration cycles increased the $\mathrm{OD}_{450}$ and falsepositivity (convex correlation) in the ELISA wells of a valid nonexpired ELISA kit. The product insert of this ELISA kit instructed to bring all reagents to room temperature each time before testing. Positive-controls, not shown, performed as expected. The expired products yielded no positive or negative reactions as expected.

deterioration of antigen binding affinity of antibodies coating the ELISA wells.

Our report highlights the importance of monitoring the appropriate performance of negative-controls to improve diagnostic accuracy, a concept that may apply to various immunoassays $[3,14]$. The way the manufacturer's instructions are interpreted by laboratory technician(s) is critical and depends on the wording and quality of the recommendations provided. At the time of testing, the Meridian ELISA product insert specifically indicated to bring to room temperature all refrigerated reagents each time prior to testing [13]. Examining the word count and content for a comparable ELISA product $[11,12]$ indicated that instructions on "storage/handling of the ELISA product" reagents compared to the instructions on "storage/handling of the fecal specimens" for testing are arguably variable and nonspecific across immunoassays $[11,13]$. Including updated technical information regarding product tolerance to repetitive refrigeration cycles and providing technical alternatives to laboratory users to minimize immunoassay exposure to unnecessary refrigeration cycles of the products would be advisable. Users would also benefit by aliquoting reagents for storage after unpacking a new product and ensuring that every ELISA test is performed with wells that have been refrigerated only once prior to use. The reduction of false-positive reactions during ELISA testing of fecal specimens, by preventing unnecessary refrigeration cycles and product deterioration, has to be accompanied by proper storage and handling of the fecal specimens $[9,15]$

\section{Conclusions}

Although the findings of false-positivity induced by repetitive refrigeration apply to the immunoassay tested, it is possible as a proof of principle to infer that the use of one negativecontrol for each ELISA run might be insufficient to monitor 
aberrant preanalytical false-positives if this or other commercial immunoassays are subject to repetitive refrigeration.

\section{Conflict of Interests}

The authors declare that there is no conflict of interests regarding the publication of this paper.

\section{References}

[1] P. Toltzis, M. M. Nerandzic, E. Saade et al., "High proportion of false-positive Clostridium difficile enzyme immunoassays for toxin A and B in pediatric patients," Infection Control and Hospital Epidemiology, vol. 33, no. 2, pp. 175-179, 2012.

[2] T. D. Wilkins and D. M. Lyerly, "Clostridium difficile testing: after 20 years, still challenging," Journal of Clinical Microbiology, vol. 41, no. 2, pp. 531-534, 2003.

[3] T. Planche, A. Aghaizu, R. Holliman et al., "Diagnosis of Clostridium difficile infection by toxin detection kits: a systematic review," The Lancet Infectious Diseases, vol. 8, no. 12, pp. 777784, 2008.

[4] M. Hanvanich, R. Viscidi, B. E. Laughon, J. G. Bartlett, and R. H. Yolken, "Stool desorbing activity: a possible cause of false-positive reactions in competitive enzyme immunoassays," Journal of Clinical Microbiology, vol. 21, no. 2, pp. 184-188, 1985.

[5] L. J. Kricka, "Interferences in immunoassay-still a threat," Clinical Chemistry, vol. 46, no. 8, pp. 1037-1038, 2000.

[6] M. Lipsitch, E. Tchetgen Tchetgen, and T. Cohen, "Negative controls: a tool for detecting confounding and bias in observational studies," Epidemiology, vol. 21, pp. 383-388, 2010.

[7] H. Martin, L. P. Abbott, D. E. Low, B. Willey, M. Mulvey, and J. S. Weese, "Genotypic investigation of Clostridium difficile in Prince Edward Island," Canadian Journal of Infectious Diseases \& Medical Microbiology, vol. 19, no. 6, pp. 409-412, 2008.

[8] H. Martin, B. Willey, D. E. Low et al., "Characterization of Clostridium difficile strains isolated from patients in Ontario, Canada, from 2004 to 2006," Journal of Clinical Microbiology, vol. 46, no. 9, pp. 2999-3004, 2008.

[9] J. Scott Weese, H. R. Staempfli, and J. F. Prescott, "Survival of Clostridium difficile and its toxins in equine feces: implications for diagnostic test selection and interpretation," Journal of Veterinary Diagnostic Investigation, vol. 12, no. 4, pp. 332-336, 2000.

[10] A. Rodriguez-Palacios, H. R. Stämpfli, T. Duffield et al., "Clostridium difficile PCR ribotypes in calves, Canada," Emerging Infectious Diseases, vol. 12, no. 11, pp. 1730-1736, 2006.

[11] Techlab, "C. DIFFICILE TOX A/B II Catalog T5015 product insert," 2014, http://www.techlab.com/wp-content/uploads/ 2013/06/t5015insert_rev_0308.pdf.

[12] S. M. Novak-Weekley and M. H. Hollingsworth, "Comparison of the premier toxin A and B assay and the TOX A/B II assay for diagnosis of Clostridium difficile infection," Clinical and Vaccine Immunology, vol. 15, no. 3, pp. 575-578, 2008.

[13] Meridian, " Premier toxin A and B catalog 616096 package insert revised 4/09," 2014, http://www.meridianbioscience.com/ Content/Assets/Files/3.1.3\%20\%20C.\%20difficile\%20Testing/ Package-Insert-Premier-Toxins-A-and-B.pdf.

[14] T. D. Planche and M. H. Wilcox, "Two-step testing for C. difficile: no answers yet," Journal of Hospital Infection, vol. 75, no. 4, pp. 325-326, 2010.
[15] J. Freeman and M. H. Wilcox, "The effects of storage conditions on viability of Clostridium difficile vegetative cells and spores and toxin activity in human faeces," Journal of Clinical Pathology, vol. 56, no. 2, pp. 126-128, 2003. 


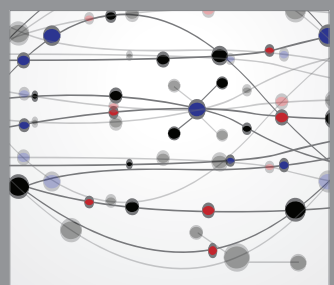

The Scientific World Journal
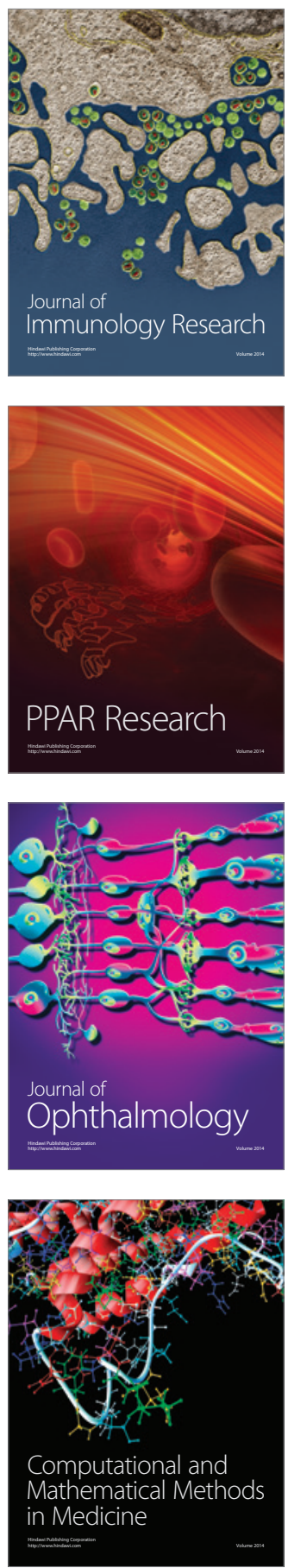

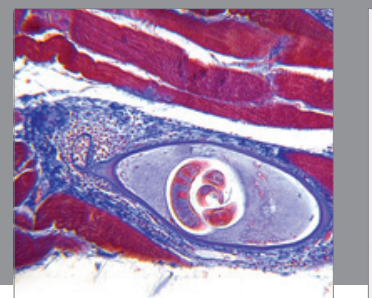

Gastroenterology

Research and Practice
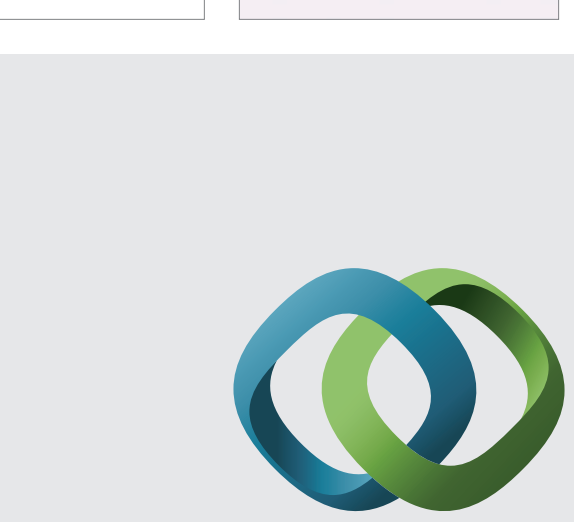

\section{Hindawi}

Submit your manuscripts at

http://www.hindawi.com
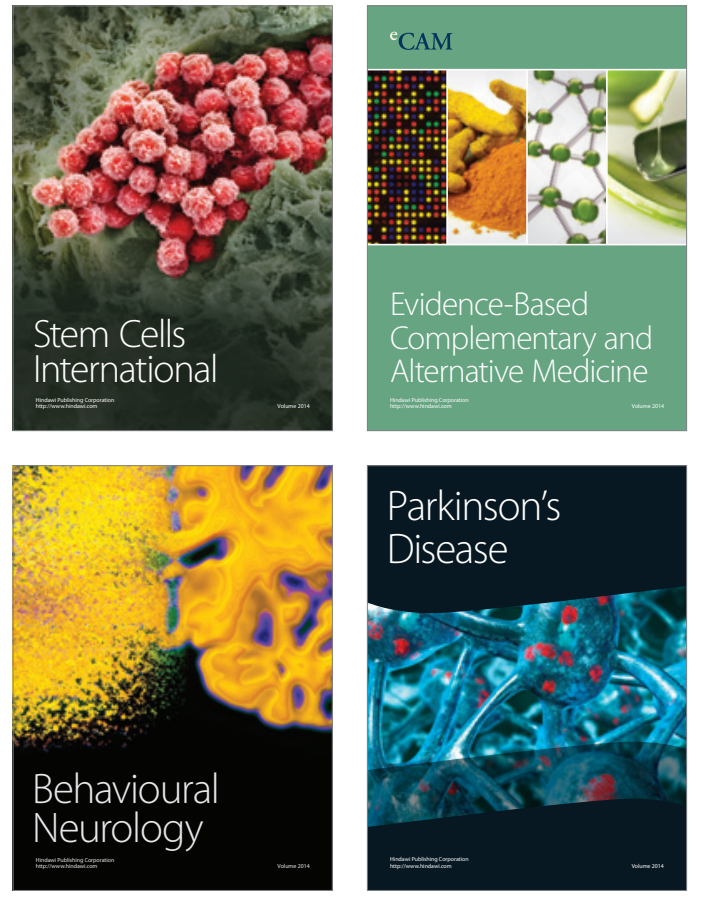
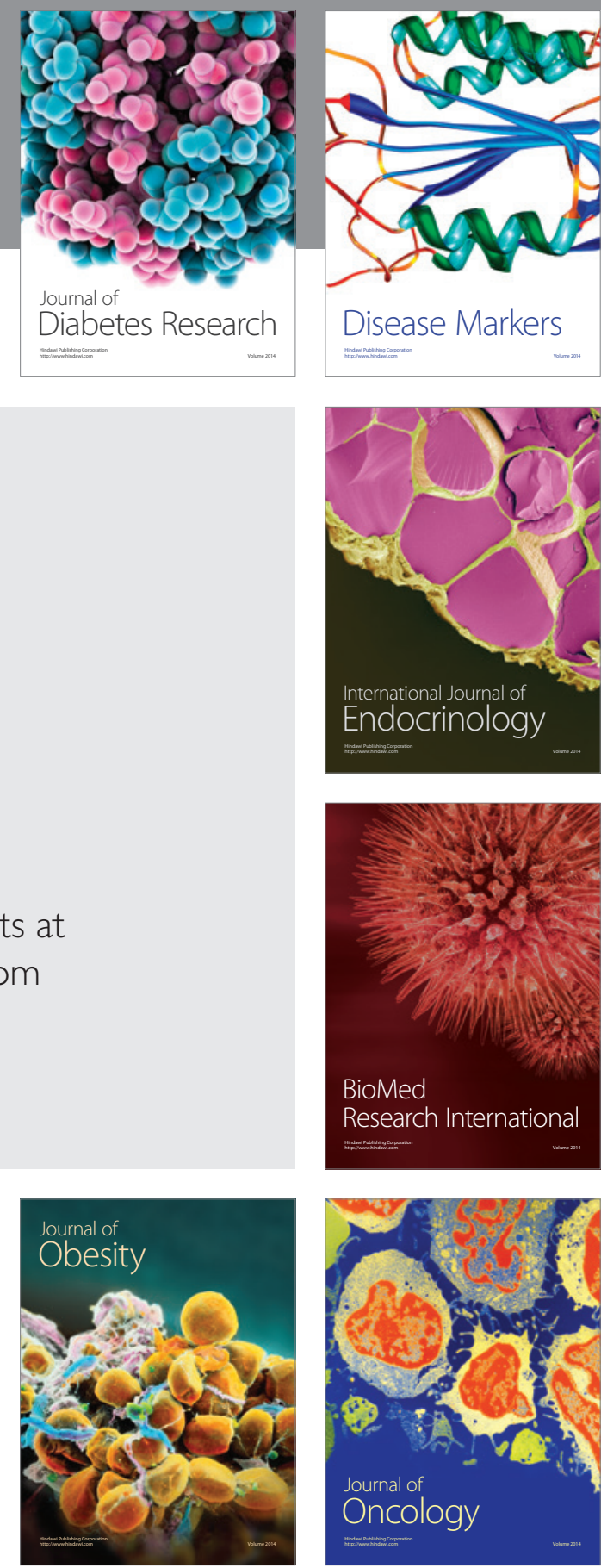

Disease Markers
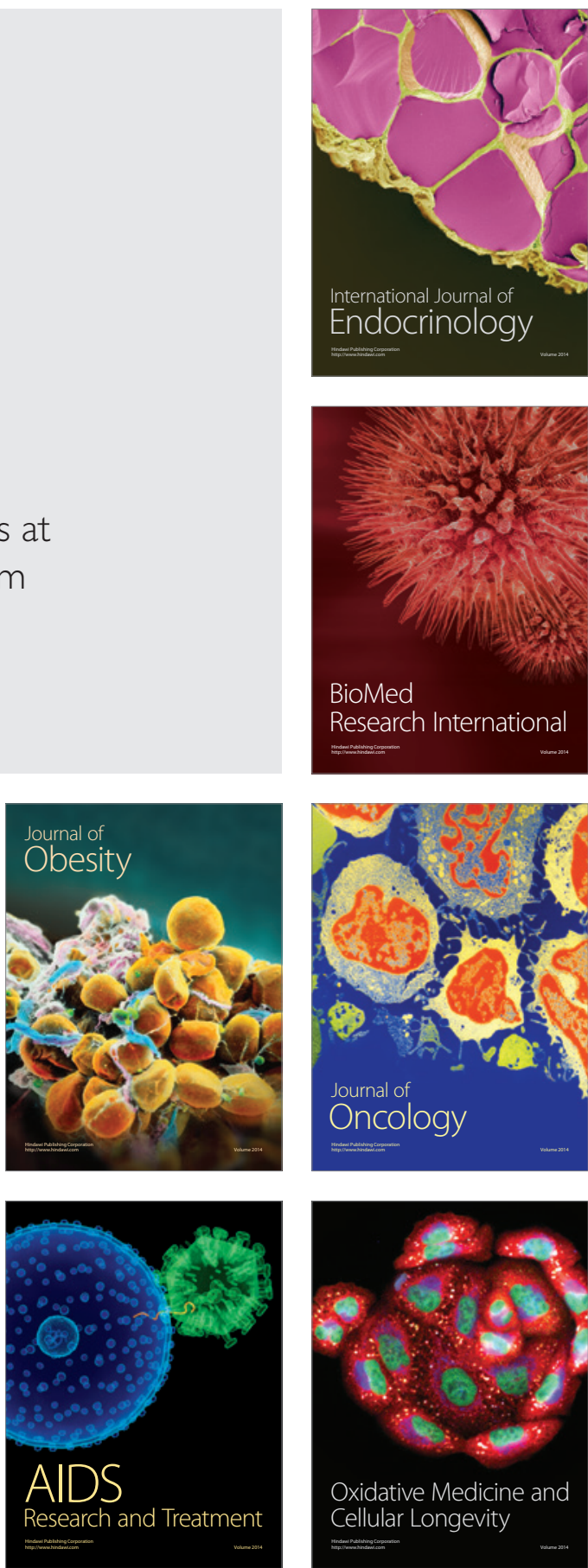